\title{
Computational Membrane Separations
}

\author{
Jianwen Jiang \\ Department of Chemical and Biomolecular Engineering, \\ National University of Singapore, 117576, Singapore
}

\begin{abstract}
Membranes are ubiquitously utilized in a wide variety of chemical separations. It is indispensable to quantitatively understand membrane microstructures and properties, as well as their effects on separation processes. With ever increasing computational power and resources, computations have become an indispensable tool and are playing an increasingly important role in membrane science and engineering.

In this presentation, the recent computational studies for a wide variety of separations in both crystalline and amorphous membranes will be discussed. For the crystalline, the focus is on metal-organic frameworks (MOFs), which provide a wealth of opportunities for engineering new membrane materials and have been considered as versatile candidates for many important applications such as $\mathrm{CO}_{2}$ capture, biofuel pervaporation and water desalination. For the amorphous, polymer membranes will be discussed for gas and liquid separations, particularly the recently emerging organic solvent nanofiltration through microporous polymer membranes.

The presentation will demonstrate that computations at an atomic/molecular level can secure the quantitative interpretation of experimental observations, provide microscopic insights from the bottom-up, and facilitate the development of new membranes.
\end{abstract}

